\section{STANDARDIZATION OF TRANSPLANT PROCUREMENT PROCEDURES}

\section{To the Editor:}

We read with interest the article by Pasque ${ }^{1}$ and applaud the author's efforts to standardize thoracic organ retrieval. Organ procurement has been conducted by surgical teams from the geographically nearest transplant center on the Zonal Retrieval Scheme in the United Kingdom since 1995. This has significantly improved cost-effectiveness and enhanced the safety of surgical teams. We recommend a number of important additions to the organ retrieval procedure that we believe to be vital in facilitating organ preservation and improving recipient outcomes.

Cardiac output monitoring (usually by using a Swan-Ganz catheter) is extremely useful, if not indispensable, in the assessment and optimization of donor hemodynamic parameters, and we strongly recommend it as a routine.

We have also found individual pulmonary vein gas sampling to be useful in recognizing unilateral dysfunction, allowing a greater number of single lungs to be identified as suitable for transplantation. ${ }^{2}$

Extension of cardiac ischemic time even beyond 1 hour progressively increases early mortality, ${ }^{3}$ and therefore minimization is vital. To this end, we routinely pack and dispatch the donor heart before completion of the donor pneumonectomy.

The author suggests extubation of the donor followed by tracheal sta-

\footnotetext{
The Editor welcomes submissions for possible publication in the Letters to the Editor section that consist of commentary on an article published in the Journal or other relevant issues. Authors should: • Include no more than 500 words of text, three authors, and five references. • Type with double-spacing. • See http://jics.ctsnetjournals.org/misc/ifora.shtml for detailed submission instructions. - Submit the letter electronically via jtcvs.editorialmanager.com. Letters commenting on an article published in the JTCVS will be considered if they are received within 6 weeks of the time the article was published. Authors of the article being commented on will be given an opportunity of offer a timely response ( 2 weeks) to the letter. Authors of letters will be notified that the letter has been received Unpublished letters cannot be returned.
}

pling. Although we appreciate the need to prevent overdistention of the donor lungs (especially with airborne transportation), we have found that complete loss of airway pressure can precipitate recollapse of previously recruited areas of atelectatic lung. Laboratory evidence suggests that both atelectasis and overdistention are detrimental to postreperfusion function. ${ }^{4}$ We therefore advocate partial inflation immediately before tracheal division. Therefore as the endotracheal tube is slowly withdrawn, the stapling device is closed and fired, allowing control of the inflation volume.

Furthermore, the inclusion of the esophagus in the lung block poses an unnecessary risk of contamination of the operative field. Dissection of the trachea from the esophagus at the time of removal of the lung block is straightforward and without injury to the airway or esophagus.

The timing of organ retrieval procedures and the regular involvement of members of staff and hospitals unfamiliar with organ retrieval can propagate many errors in acquiring accurate information and ensuring full and complete communication. Procedural errors are also possible in such a complex and time-sensitive undertaking. We feel strongly that such errors could be avoided by the use of an appropriate procedural checklist for both retrieval and implantation surgical teams. ${ }^{5}$ An adaptation of the World Health Organization's surgical checklist has recently been adopted in the United Kingdom, and cardiopulmonary transplantspecific checklists might be of great value in ensuring best practice.

We once again congratulate Dr Pasque on an excellent and timely synopsis but believe that our supplementary points can further enhance patient safety and improve outcomes.

Phil Botha, MRCS

Stephen C. Clark, FRCS (Cth) John H. Dark, FRCS

Department of Cardiopulmonary Transplantation

\section{Freeman Hospital \\ Newcastle upon Tyne, United \\ Kingdom}

\section{References}

1. Pasque MK. Standardizing thoracic organ procurement for transplantation. $J$ Thorac Cardiovasc Surg. 2010;139:13-7.

2. Aziz TM, El-Gamel A, Saad RA, Migliore M, Campbell CS, Yonan NA. Pulmonary vein gas analysis for assessing donor lung function. Ann Thorac Surg. 2002;73:1599-604.

3. Banner NR, Thomas HL, Curnow E, Hussey JC, Rogers CA, Bonser RS. The importance of cold and warm cardiac ischemia for survival after heart transplantation. Transplantation. 2008;86:542-7.

4. DeCampos KN, Keshavjee S, Liu M, Slutsky AS. Optimal inflation volume for hypothermic preservation of rat lungs. J Heart Lung Transplant. 1998; 17:599-607.

5. Haynes AB, Weiser TG, Berry WR, Lipsitz SR, Breizat AH, Dellinger EP, et al. A surgical safety checklist to reduce morbidity and mortality in a global population. $N$ Engl J Med. 2009;360:491-9.

doi:10.1016/j.jtcvs.2010.03.027

\section{Reply to the Editor:}

The stated goal of the publication of a proposed standardization of the thoracic organ procurement protocol was to stimulate enlightened commentary from leading international thoracic transplant centers, such as that found at the Freeman Hospital in Newcastle upon Tyne, United Kingdom. It is only with the input from such centers of excellence that the protocol can be optimized. Although the final adoption of a protocol on which everyone agrees is unlikely, the completion of this vital open-forum dialogue between centers at the very least educates the next generation of surgeons in regard to the major issues and the available technical options.

Although we have not found it necessary to insist on the placement of a pulmonary artery catheter in the overwhelming majority of cases as suggested by the Newcastle group, we have no problem with its routine application and most certainly have found it to be of critical importance in high-risk donors in whom optimal hemodynamic management or organ use decisions are in need of more quantified hemodynamic information. A best estimate of our current use of 